This decision, which enables the Lithuanian Red Cross Socicty to resume its place within the International Red Cross and Red Crescent Movement. brings the number of duly recognized National Societies to 149.

\title{
Recognition of the Latvian Red Cross Society confirmed
}

The International Committee of the Red Cross confirmed on 20 November 1991 the validity of its recognition of the Latvian Red Cross Society announced on 10 January 1923.

This decision, which enables the Latvian Red Cross to resume its place in the International Red Cross and Red Crescent Movement, brings to $\mathbf{1 5 0}$ the number of duly recognized National Societies. 